\title{
Nature of the charge localized between alkali adatoms and metal substrates
}

\author{
G. K. Wertheim, D. M. Riffe, ${ }^{*}$ and P. H. Citrin \\ AT\&T Bell Laboratories, Murray Hill, New Jersey 07974
}

(Received 20 October 1993)

\begin{abstract}
Two previously unappreciated features in photoemission spectra from alkali atoms adsorbed on W(110), namely, the sign of the alkali-induced surface-atom core-level shift of the substrate at low coverage and the very large alkali shallow core-hole lifetime width at all coverages, show that the alkalisubstrate interaction is not well described by a transfer of alkali charge. Instead, both features point to the formation of a charge cloud between the alkali adatom and substrate that is derived largely from alkali valence states.
\end{abstract}

\section{INTRODUCTION}

Some years ago, we argued that the Langmuir-Gurney model of alkali adsorption ${ }^{1,2}$ could not account for either substrate or adsorbate photoemission spectra obtained from alkali-covered $\mathrm{W}(110)$ surfaces. $^{3}$ The essential features of this model are, at low coverage, the creation of an inverted surface dipole by donation of charge from the alkali adatoms into the substrate conduction band, followed at high coverage by a reverse donation of charge from the substrate atoms back to the alkali adatoms. (Such "back donation" is needed to explain the coverage dependence of the work function.) One aspect of this model, namely, that close to saturation, most of the alkali charge is in a metallic band made up of alkali orbitals, was confirmed by band-structure calculations for $\mathrm{Cs}$ on $\mathrm{W}(100){ }^{4}$ More recently, various calculations have shown that there is a well-localized cloud of charge situated between the adsorbate and substrate atoms at all coverages. ${ }^{5,6}$ Significantly, however, one group ${ }^{6}$ arrives at this conclusion starting with the ansatz that there is essentially complete charge transfer, while another ${ }^{5}$ finds that the alkali adatoms remain essentially neutral.

In large measure, the controversy regarding the alkalisubstrate interaction boils down to the question: What is the nature of this charge cloud? If it is made up principally of substrate orbitals, the concept of charge transfer can be justifiably invoked; if it is made up largely of adsorbate orbitals, it cannot. As in most controversies, there seems to be evidence supporting both views. For example, an attempt has been made using first-principles density-functional calculations to show that alkali orbitals are not involved, arguing that the substrate charge screening a bare, fractional test charge appears similar to that of a single adsorbed alkali atom. ${ }^{6}$ However, earlier work demonstrates convincingly that alkali $s$ states are always at least partially occupied. Gurney ${ }^{2}$ pointed out that the interaction of the alkali $s$ orbital with the metallic conduction band necessarily broadens the $s$ level into a resonance and that the position of the resonance determines the occupation of the alkali $s$ level. Later theoretical work focused on solving the Gurney picture using various model calculations. ${ }^{7-10}$ Muscat and Newns ${ }^{8,9}$ introduced the concept of intra-atomic $s-p$ hybridization and polarization to account for the properties of the adsorbate system, while Ishida and Terakura ${ }^{5}$ (IT) concluded that the adsorbate atoms are neutral at all coverages and that the inverted dipole arises from hybridization of the alkali $s$ level with substrate orbitals. Finally, Scheffler et al. ${ }^{6}$ have taken the position that it is simply sufficient to know what the spatial charge distribution is, dismissing altogether the controversy over the nature of the interaction as one of semantics.

In this work, we examine whether photoemission data from adsorbate and substrate atoms can provide any constraints on the interpretation of this localized charge cloud. A detailed study is presented of the properties of Cs on W(110), focusing primarily on the line shape of the Cs $5 p$ core-level spectra as a function of coverage. It is shown that both the Lorentzian lifetime width and the singularity index do provide information about the nature of the charge associated with the alkali adsorbate atoms. In addition, a more detailed account is presented concerning the effect of adsorbed alkali atoms on the sign and magnitude of the substrate surface-atom core-level binding energy, from which a well-defined and unexpected coverage dependence is revealed. The sum of these results leads to the conclusion that the charge cloud formed between the alkali adsorbate and the substrate does indeed have a significant amount of alkali $s$ character, even at low coverage. Recent arguments attempting to retain the notion of alkali charge transfer are critically examined.

\section{RESULTS}

\section{A. Alkali-adatom core-electron binding energy}

Sample preparation and data acquisition have been described in Ref. 3. For simplicity here, we focus mainly on the results from Cs because the spin-orbit splitting of the outermost $p$ level is sufficiently large to analyze the $5 p_{3 / 2}$ spectrum without interference from the $5 p_{1 / 2}$ component. Similar findings were obtained for $\mathrm{Na} 2 p$ and $\mathrm{K}$ $3 p$ core levels and are mentioned where appropriate.

Data for the Cs $5 p$ photoemission spectrum on a $\mathrm{W}(110)$ substrate, shown in Fig. 1, exhibit trends that are similar to those reported previously for alkali metals on 


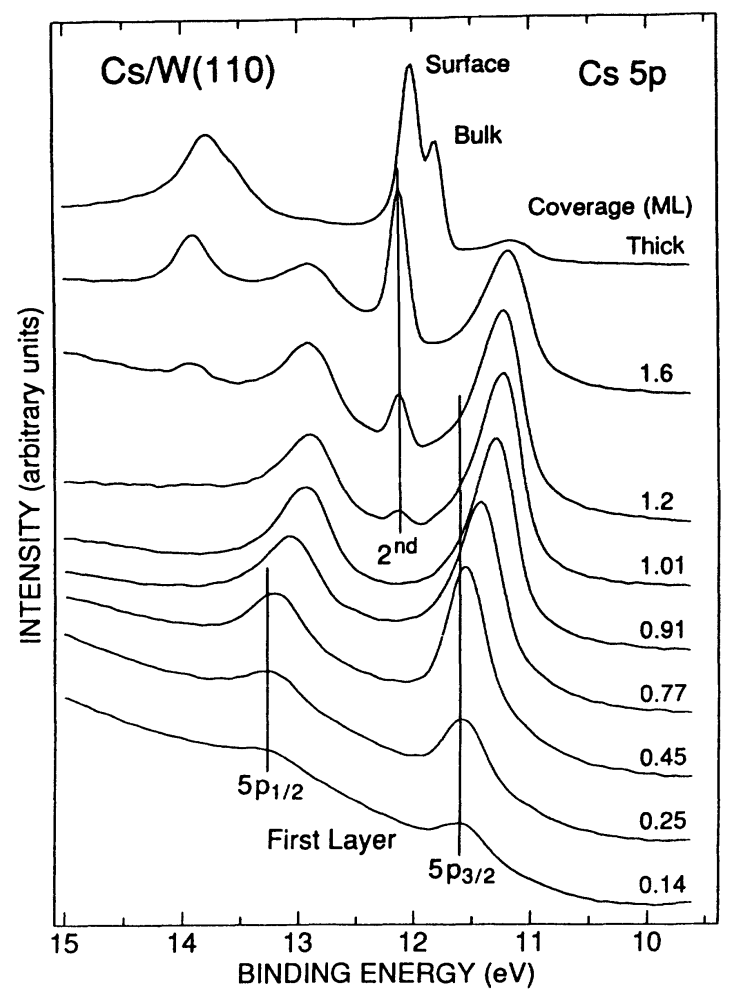

FIG. 1. Cs $5 p$ photoemission spectra for Cs deposited on $\mathrm{W}(110)$ at $78 \mathrm{~K}$. The photon energy is $29.5 \mathrm{eV}$. Coverage is normalized to first-layer saturation, defined here as 1.0 monolayer (ML).

other metal substrates. ${ }^{11-13}$ The $5 p$ binding energy in the first atomic layer is observed to decrease, first slowly at low coverage while the adatoms are well separated, and then more rapidly at higher coverage, when they begin to interact. Both dipolar repulsion and band formation $^{4,5,7-9}$ are responsible for this behavior. Atoms in the second layer have a smaller binding energy than those in the first layer and desorb more readily, consistent with the Cs-Cs interaction being weaker than the Cs-substrate interaction. There is no change in the second-layer binding energy with coverage, indicating there is no dipole moment associated with these atoms. The other notable feature, discussed in Sec. II B, is that the second-layer spectrum is much narrower than that of the first. When a thicker layer is deposited, the spectrum exhibits the two-peak structure similar to that observed in the study of bulk Cs. However, the first-layer signal still remains visible, demonstrating that such an overlayer is not uniform and probably consists of islands or clusters. The surface component of this thick layer has a slightly larger binding energy than that of the bulk, but it is still smaller than that of the second layer. This indicates that the interaction of the Cs directly in contact with the W(110) substrate is so strong and distinctly different than that of any other Cs-Cs interaction that its effect can still be seen to persist in the second layer.

The above description of the behavior of Cs $5 p$ on $\mathrm{W}(110)$ applies in every detail to the data for $\mathrm{K} 3 p$ on $\mathrm{W}(110)$; see Fig. 2. The only difference is that the $\mathrm{K} 3 p$

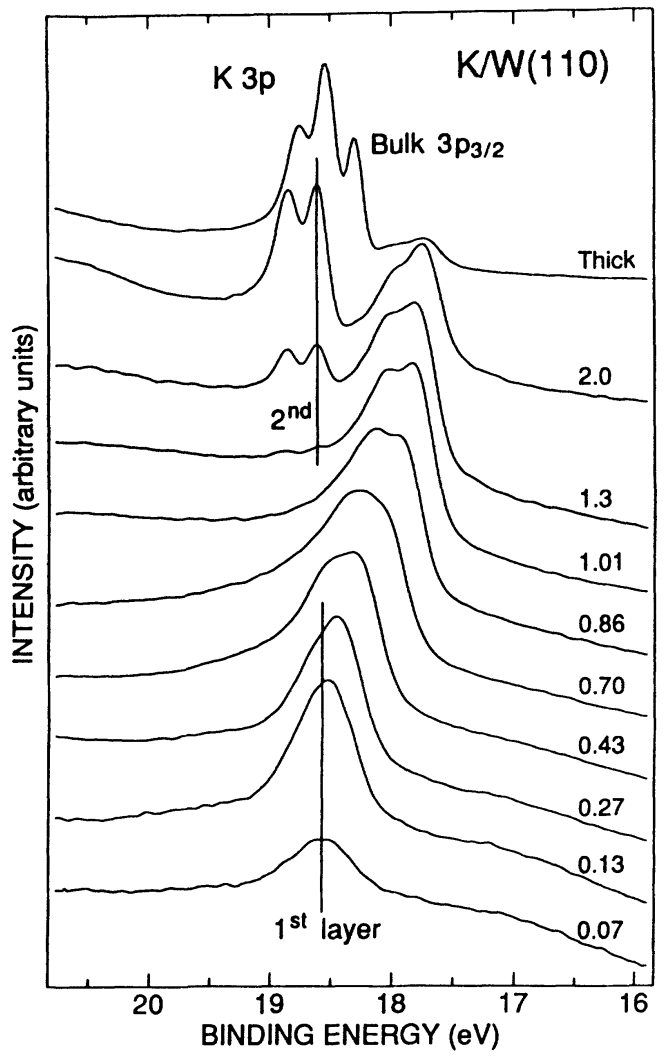

FIG. 2. K $3 p$ photoemission spectra for Cs deposited on $\mathrm{W}(110)$ at $78 \mathrm{~K}$. The photon energy is $41 \mathrm{eV}$. Coverage is normalized to first-layer saturation, defined here as $1.0 \mathrm{ML}$.

spin-orbit splitting is much smaller than that of Cs $5 p$, so the first-layer components are poorly resolved. The fact that the second-layer spectrum is again much narrower than the first-layer spectrum is apparent in the wellresolved spin-orbit doublet obtained from that layer. The thick-layer spectrum exhibits three peaks because the surface-atom core-level shift is comparable to the spinorbit splitting. As mentioned above, all these data are typical for alkali-adsorbate systems.

Since the behavior of the alkali adlayer in contact with the metal substrate has been the principal subject of both theoretical and experimental work in recent years, the large and easily measurable change in surface-alkali-atom core-level binding energy with coverage is the feature that initially attracts attention when the photoemission data are examined. Unfortunately, it does not yield significant insight into the electronic nature of the localized charge cloud, because all mechanisms - back donation, dipolar repulsion, and band formation-imply a decrease in binding energy with increasing coverage. For this reason, we look to other features in the data.

\section{B. Alkali-adatom linewidth}

At least as striking as the larger binding-energy change with coverage is the very large core-level linewidth associated with the adsorbed alkali layer in direct contact with the substrate. This broadening is not unique to Cs on W(110), but can be generally seen in high-resolution 
data from several other systems, e.g., $\mathrm{Na} / \mathrm{W}(110)$ and $\mathrm{K} / \mathrm{W}(110),{ }^{3} \mathrm{Cs} / \mathrm{Pt}(111),{ }^{11} \mathrm{Cs} / \mathrm{Ru}(100),{ }^{12}$ and $\mathrm{Cs} / \mathrm{Al}(111) .^{13}$ We illustrate this in Fig. 3, including for comparison data from bulk Cs and, as reported in Ref. 13, data from Cs on Al(111). The full width at half maximum of the Cs $5 p_{3 / 2}$ data from the saturated layer on $\mathrm{W}(110)$ is about $500 \mathrm{meV}$.

The anomalous width of this shallow adatom core level could be due to a combination of mechanisms, including phonon and inhomogeneous broadening of Gaussian shape and lifetime broadening of Lorentzian shape. To determine quantitatively the shape and source of this broadening requires fitting the set of data, covering the range $0-1$ monolayers (ML), ${ }^{14}$ with Doniach-Šunjić (DS) lines ${ }^{15}$ convolved with Gaussian functions. The Lorentzian width of the $5 p$ spin-orbit components were free parameters, but the singularity index and Gaussian width were constrained to the same value for both components. The secondary-electron-dominated background used in the analysis was obtained from spectra covering the same range of kinetic energy but taken with higher photon energy. The results of the analysis for two representative coverages are shown in Fig. 4. (The background is suppressed in the figure to facilitate comparison of the line shapes.) The analysis shows that the broadening is dominated by Lorentzian character, i.e., that it is due to a greatly reduced core-hole lifetime. In addition, the Gaussian width is larger than in bulk Cs.

The Gaussian width, representing instrumental, phonon, and inhomogeneous contributions, varies consider-

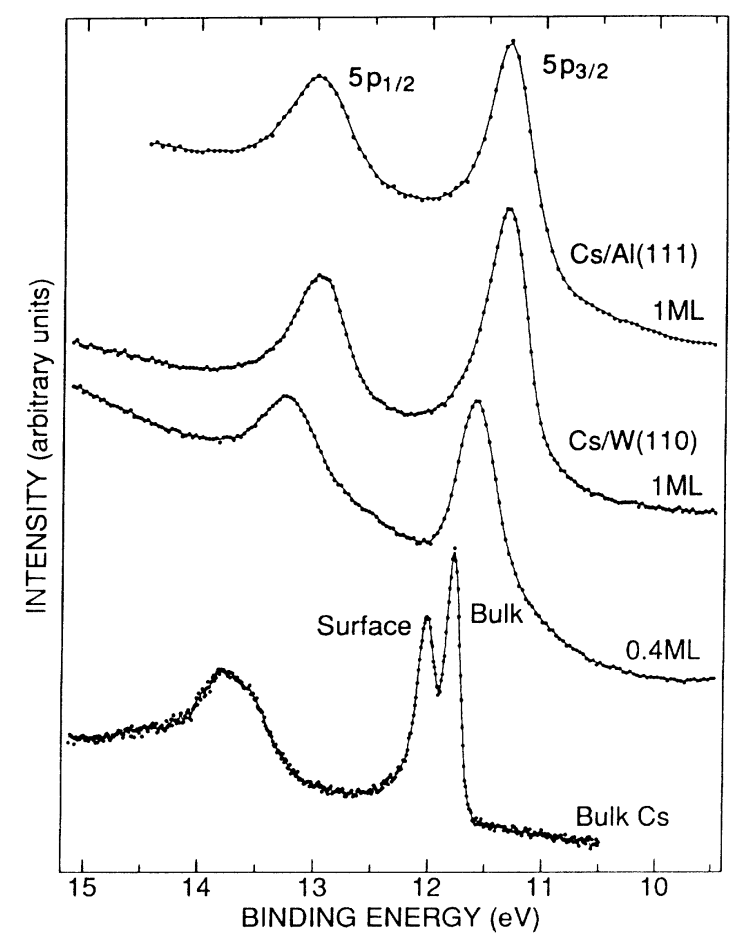

FIG. 3. Cs $5 p$ photoemission spectra of Cs adsorbed on $\mathrm{W}(110)$ and $\mathrm{Al}(111)$ compared with the spectrum of bulk Cs. The Al substrate data are from Ref. 13.

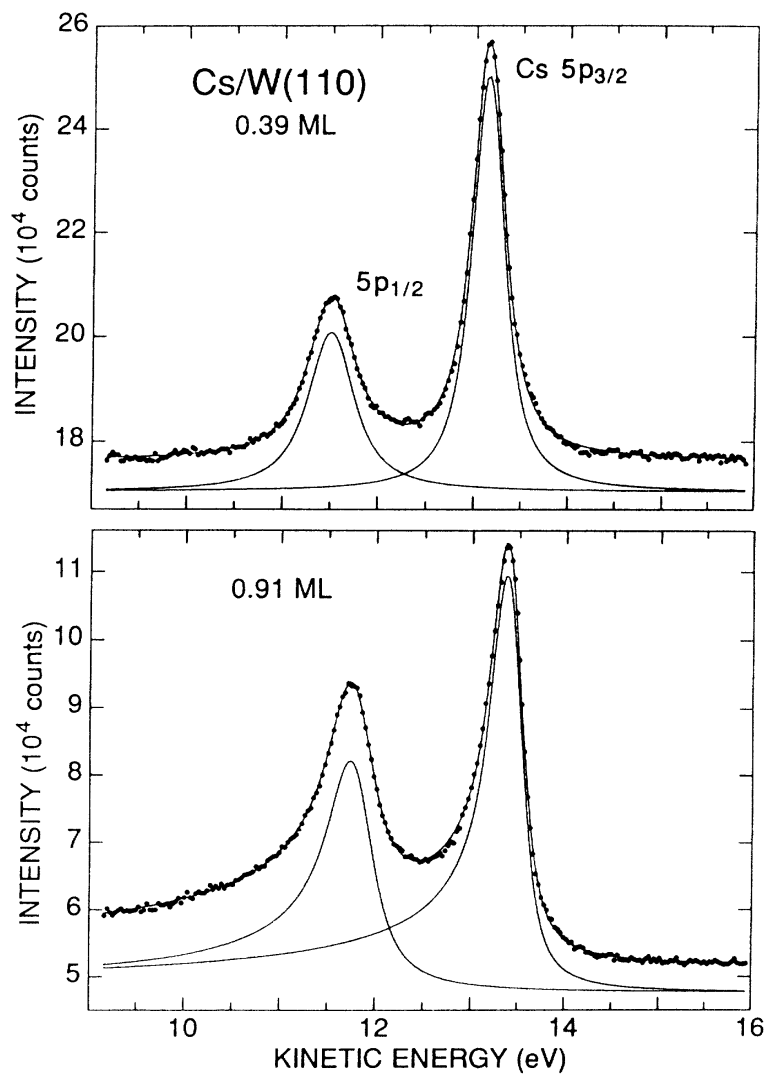

FIG. 4. Least-squares fit to photoemission spectra of firstlayer Cs adsorbed on W(110) at low coverage (top) and at high coverage (bottom). Background has been suppressed to emphasize difference in line shape.

ably with coverage; see Fig. 5. The instrumental broadening of $\sim 85 \mathrm{meV}$ makes only a minor contribution. The particularly large value at low coverage, 265 $\mathrm{meV}$, reflects the weak corrugation of the in-plane potential that allows the Cs to diffuse rapidly on the surface even at $78 \mathrm{~K}$. This is consistent with the absence of an observable low-energy electron diffraction (LEED) pattern associated with the overlayer for coverages less than $0.25 \mathrm{ML}^{16}$ Between $\sim 0.4$ and $0.6 \mathrm{ML}$, we see a clear ring pattern, while above $\sim 0.8 \mathrm{ML}$ a hexagonal LEED pattern emerges. The variations of the total Gaussian width seen in Fig. 5 correlate well with our observed LEED changes. It is clear, therefore, that this width bears mainly on the intralayer dynamics and structure of the adsorbate atoms and contains little information directly related to the alkali-substrate interaction.

The Lorentzian width, on the other hand, reflects the rate of an Auger decay process involving the $6 \mathrm{~s}$ electron and thus probes the electronic environment of the Cs atom in a way directly relevant to the nature of the alkali-adatom-metal-substrate interaction. The numerical results for the $5 p_{3 / 2}$ lifetime width are plotted in Fig. 5. By contrast with the nonmonotonic variations of the Gaussian width observed throughout the full coverage range, the Lorentzian width is relatively unchanged up until $\sim 0.5 \mathrm{ML}$, after which it steadily decreases as satu- 
ration coverage is approached. We first consider the source of the enormous increase of the Cs $5 p_{3 / 2}$ lifetime width in the adsorbed state at low coverage relative to that in the bulk metal. Below $0.45 \mathrm{ML}$, this width is
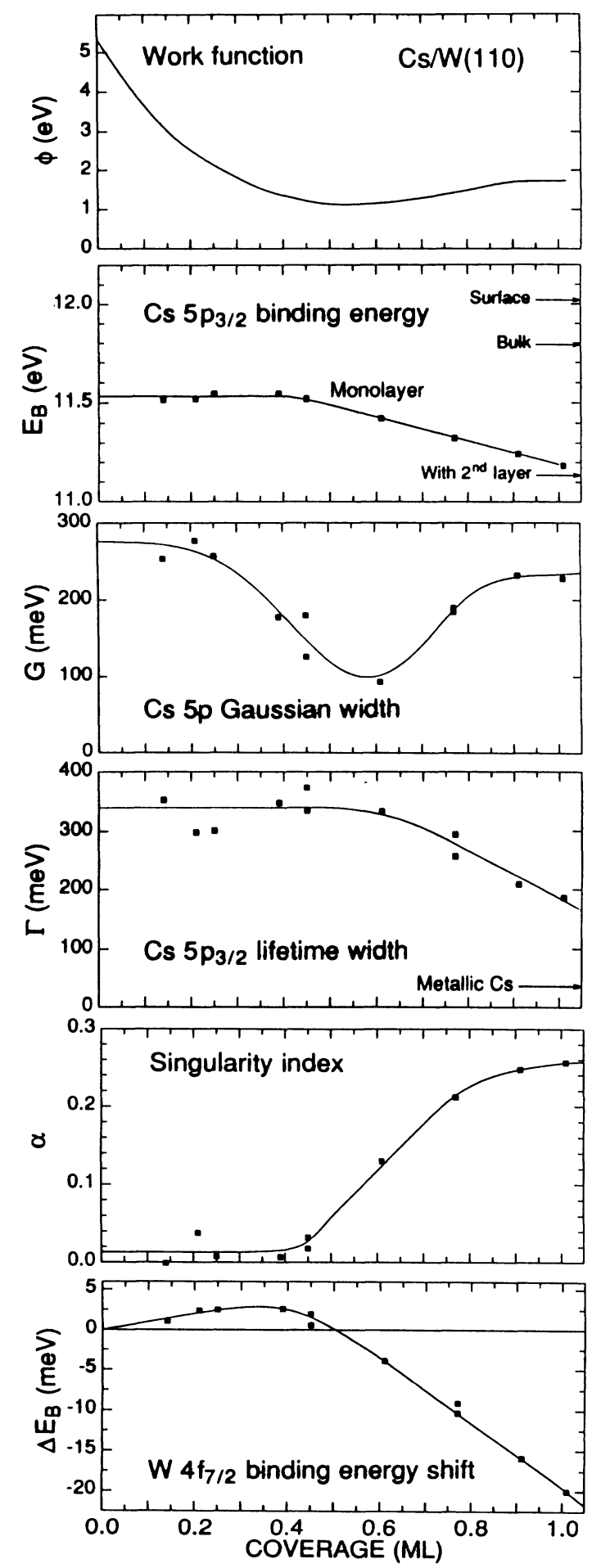

FIG. 5. Work function of Cs-covered W(110) from Ref. 16, Cs $5 p_{3 / 2}$ binding energy, Gaussian width, lifetime width, singularity index, and relative change in $\mathbf{W ~} 4 f_{7 / 2}$ surface-atom corelevel shift, all plotted as a function of coverage. Here 1.0 ML is defined as saturation coverage. All solid lines drawn through the data points are guides to eye.
$340 \pm 40 \mathrm{meV}$, approximately ten times larger than the bulk-metal lifetime width of $35 \pm 8 \mathrm{meV} .^{17}$ To our knowledge, this represents the largest identified change in core-level lifetime width produced by changes in the chemical environment. Data for $\mathrm{Na}$ and $\mathrm{K}$ on $\mathrm{W}(110)$ show a comparable enhancement of Lorentzian widths for the corresponding outermost core levels. For $\mathrm{K} / \mathrm{W}(110)$, the $3 p$ lifetime width at low coverage is $\sim 250$ meV compared with $14 \pm 4 \mathrm{meV}$ (Ref. 18) in the bulk metal, while for $\mathrm{Na} / \mathrm{W}(110)$ the high-coverage $2 p$ width is $115 \mathrm{meV}$ compared with $10.5 \pm 1 \mathrm{meV}$ (Ref. 19) in the metal. An earlier explanation ${ }^{12}$ of the large $5 p_{3 / 2}$ linewidth for Cs on $\mathrm{Ru}(111)$, involving partial filling of the normally empty $5 d$ band which facilitates a $\mathrm{O}_{3} \mathrm{O}_{4,5} \mathrm{P}_{1}$ Coster-Kronig decay, cannot account for the comparably enhanced $\mathrm{Na} 2 p_{3 / 2}$ width because an analogous decay channel does not exist in $\mathrm{Na}$.

The key to understanding the large lifetime width of the shallowest alkali-adatom $p_{3 / 2}$ hole state lies in the nature of the alkali-substrate electronic interaction. The decay of this shallow core hole in the bulk metal is due almost exclusively to the core-valence-valence Auger process $^{20}$ which is forbidden in the free alkali atom containing only one valence $s$ electron. In the metal, the second valence electron is supplied by the predominantly $s$-like conduction band in response to screening the outermost $p$ hole state.

With this understanding, it should now be apparent that the large lifetime width in adsorbate atoms is fully at odds with the simple Langmuir model of adsorption. In this picture, the alkali valence orbitals are essentially empty in the ground state. As a result, there is only one electron in adsorbate valence orbitals in the photoemission final state (due to the charge-transfer process that screens the core hole $\left.{ }^{10,21}\right)$, thereby precluding conventional Auger decay. Only an interatomic Auger process, in which the second electron is supplied by the substrate, is feasible, but this process is too slow ${ }^{22}$ to account for the very large lifetime width observed here. Even the Langmuir-Gurney model, ${ }^{1,2}$ in which the alkali $s$ resonance is partially occupied at all coverages, is incompatible with the data because back donation would increase the lifetime width with increasing coverage, contrary to what is observed. It follows, therefore, that one valence electron must already be present in the ground state of the alkali adatom, so that the screened hole state has the requisite two electrons in adsorbate valence levels to accommodate the Auger decay.

The above arguments establish that substantial occupancy of alkali valence states is a necessary condition for the Auger process to occur, but it is not sufficient to account for the significantly increased Auger decay rates found in the adsorbate atoms. The additional factor is found in the orbital character of the alkali valence charge. Calculated charge-density contours for alkali adatoms $^{5,6}$ show that they are highly distorted relative to those of an alkali atom, indicating that the polarized valence charge contains an admixture of higher angular momentum states. In short, the s level is hybridized. Substantial $s-p_{z}$ mixing is expected because of polarization along the surface normal and because the $n p$ levels of the 
free alkali atoms are relatively close in energy to the $n s$ levels, e.g., $1.44 \mathrm{eV}$ for $\mathrm{Cs}$ and $2.10 \mathrm{eV}$ for $\mathrm{Na}$. The resulting occupied $p_{z}$ character has a profound effect on the core-hole lifetime. While there are no calculations for $\mathrm{Na}$ itself, the results for $\mathrm{Al}$ and $\mathrm{Si}$ (Ref. 23) show that the Auger rate with a $3 s 3 p$ outer shell configuration is about an order of magnitude larger than that for a $3 s^{2}$ outer shell. The very small amount of $p$ character in the occupied part of an alkali-metal conduction band is thus consistent with the much smaller lifetime width found there. It is interesting to note that an enhanced $3 p$ lifetime width has also been observed in data for $\mathrm{K} / \mathrm{Si}(100),{ }^{24}$ where the alkali atoms occupy well-defined adsorption sites. Since the bonding there can be described with more confidence in terms of alkali-adatom $s-p$ hybridization, the interpretation given above applies to semiconducting substrates as well. Of course, the concept of valence $s-p_{z}$ hybridization in alkali atoms (internal polarization) is not new, having been considered both in earlier model calculations ${ }^{8,9}$ and in more recent theoretical work. ${ }^{5}$ What has not been appreciated is its impact on the core-hole lifetime.

It might be argued at this point that the large Auger rate associated with $\mathrm{Cs}$ adatoms indicates only that the overlap between an outer-shell $5 p$ hole and the charge cloud located between the alkali atom and the substrate is comparable to that between the $5 p$ hole and electrons in the $6 s$ and $6 p$ shells, i.e., that the observed effect on the lifetime width does not prove that the charge cloud is made up of alkali orbitals. To assess whether a charge cloud comprised mainly of substrate orbitals might instead be responsible for this very effective Auger process would require a calculation of the Auger matrix elements involving the theoretical charge distribution. This is a formidable but not impossible task and could help to resolve the question about the nature of this charge cloud. However, in view of the fact that several approaches $^{2,5,8,9}$ already show the alkali $s$ resonance to be partially occupied even at low coverage, and the general agreement that an alkali $s$ band is formed at high coverage, coupled with the evidence cited above arguing against back donation, it seems more likely that the ground-state charge does reside in adsorbate-derived orbitals at small coverage as well.

The decrease in the lifetime width at high coverage emerges naturally from the depolarization associated with the formation of the alkali $s$ conduction band, which results in a weakening of the $s-p$ hybridization. The lack of a basic change in the character of the charge cloud as the coverage varies is consistent with the theory of IT. ${ }^{5}$

\section{Alkali-adatom singularity index}

The two different Cs coverages on $\mathrm{W}(110)$ in Fig. 4 illustrate another important property of adsorbed alkali layers. The line shape is observed to change from being symmetric at low coverage to asymmetric at high coverage, with a long tail toward higher binding energy. As shown in Fig. 4, the asymmetrical lines are well fitted with the DS line shape associated with the many-body screening response of a metallic conduction band. ${ }^{15}$ The analysis of the Cs $5 p$ data yield the singularity indices shown in Fig. 5. The essentially symmetrical lines at low coverage indicate there is no measurable many-body screening response from the conduction electrons of the substrate. Instead, as calculated by Lang and Williams, ${ }^{21}$ the adsorbate core hole is screened by transfer of charge into the outer $s$ level. The singularity index starts to become measurable at a coverage of about $0.45 \mathrm{ML}$ and reaches a value of 0.26 at saturation. It is worth noting that the onset of intralayer metallic screening does not result in a discontinuity in the Cs $5 p$ core-electron binding energy. The only effect is the beginning of a small decrease in the binding energy as the singularity index increases, consistent with a many-body screening response from electrons within the alkali $s$ conduction band being more effective than a screening response coming from the transfer of substrate electrons into an outer alkali orbital.

The saturation value of the singularity index for Cs is substantial and is in fact somewhat larger than that associated with surface atoms of bulk alkali metals. ${ }^{25}$ This is consistent with the observation that the singularity index increases with increasing amount of $s$-wave screening charge. ${ }^{26}$ Thus, the surface component has a larger value than the bulk because the narrowed surface density of states is clearly more atomiclike, i.e., contains a greater degree of $s$-like character. The alkali adlayer at higher coverage, in turn, is even more atomiclike. This localization of $s$-like screening charge in the final state more than compensates for the admixture of alkali $p$ character in the initial state (the $s-p$ hybridization is, as mentioned above, less important at higher coverages). It is significant to note that the large observed singularity index would be very difficult to understand if the alkali adlayer conduction band were made up of substrate orbitals, because contributions of screening charge with $p$ and $d$ character would lead to a much smaller value. ${ }^{26}$ Since the only apparent change with coverage seen in the charge density maps of IT (Ref. 5) is due to a change in the degree of overlap between identical regions of adatom screening charge, there is no reason to assume that the charge resides in anything but adatom states at all coverages.

As an aside, we note that the singularity index for Cs on an $\mathrm{Al}(111)$ substrate $^{13}$ is found to be 0.24 at saturation, comparable to that for Cs on W. This further supports the fact that the substrate metal conduction band does not make a major contribution to the screening process at high coverage, i.e., the strong many-body response is due almost entirely to the adsorbate conduction band. Highquality data at low coverage would be desirable for studying the screening transition in this system as well.

\section{Alkali-induced W $4 f$ surface-atom core-level shift}

In general, interpreting photoemission spectra from metal surface atoms is more straightforward than interpreting spectra from adsorbates, because changes in final-state screening with coverage are much smaller (the substrate surface atoms are already well screened by the bulk conduction electrons ${ }^{27}$ ) and because the electronic configuration of the adsorbate is minimally perturbed by 
substrate-atom photoionization. Accordingly, such spectra are better suited for monitoring the effects of initialstate adsorption without significant distortion from the final-state core hole.

We had reported earlier ${ }^{3}$ that Cs coverages of $\frac{1}{3}$ and full saturation on $W(110)$ lead, respectively, to shifts of only $+3 \pm 2$ and $-20 \pm 3 \mathrm{meV}$ in the $\mathrm{W} 4 f$ surface-atom core-electron binding energy $\Delta_{s, b}$. Shifts of such magnitude are very small compared with the value of $\Delta_{s, b}$ itself for the clean surface, $321 \pm 2 \mathrm{meV}$, and with the shifts induced by the adsorption of hydrogen ${ }^{28}$ or oxygen. ${ }^{29}$ More detailed data for $\mathrm{Cs}$ on the $\mathrm{W}(110)$ surface are shown in Fig. 6, where small but systematic changes in the $4 f_{7 / 2}$ surface-atom core-level with coverage are observed. Analogous data for $\mathrm{Na}$ on $\mathrm{W}(110)$ exhibit similar behavior; see Fig. 7. Comparably small shifts are also produced by the deposition of alkali metals on $\mathrm{Ta}(110){ }^{30}$

Changes in the average $\Delta_{s, b}$ for $\mathrm{W}(110)$, obtained by fitting all the core-level data for adsorbed $\mathrm{Na}, \mathrm{K}$, and $\mathrm{Cs}$, are shown in Fig. 8. Note that in every case at low coverage, the shift is initially toward larger binding energy, i.e., opposite to that expected for charge donation to the substrate. The effect on those substrate atoms directly at a site occupied by an alkali adsorbate atom is obtained by dividing the average values of the shifts by the fractional coverage, yielding +5 , +23 , and $+8 \mathrm{meV}$ for $\mathrm{Na}, \mathrm{K}$, and $\mathrm{Cs}$, respectively (all $\pm 2 \mathrm{meV}$ ). The corresponding shifts for a $\mathrm{Ta}(110)$ substrate are considerably larger,

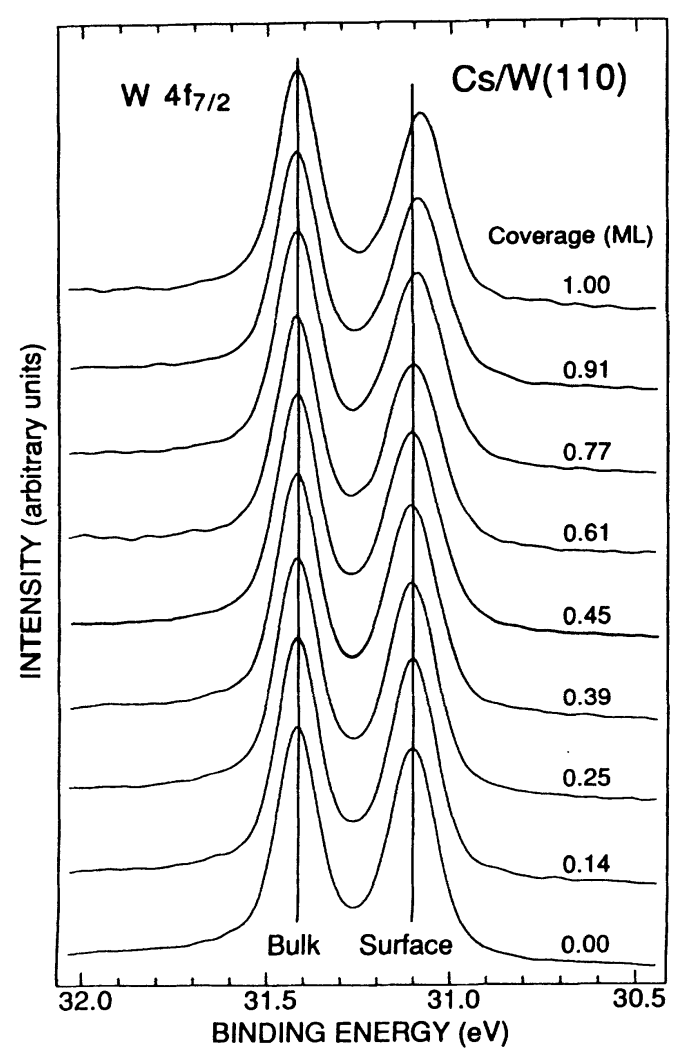

FIG. 6. W $4 f_{7 / 2}$ photoemission spectra from $\mathrm{W}(110)$ at $78 \mathrm{~K}$ adsorbed with Cs. Coverage is given as a fraction of saturation, defined here as $1.0 \mathrm{ML}$.

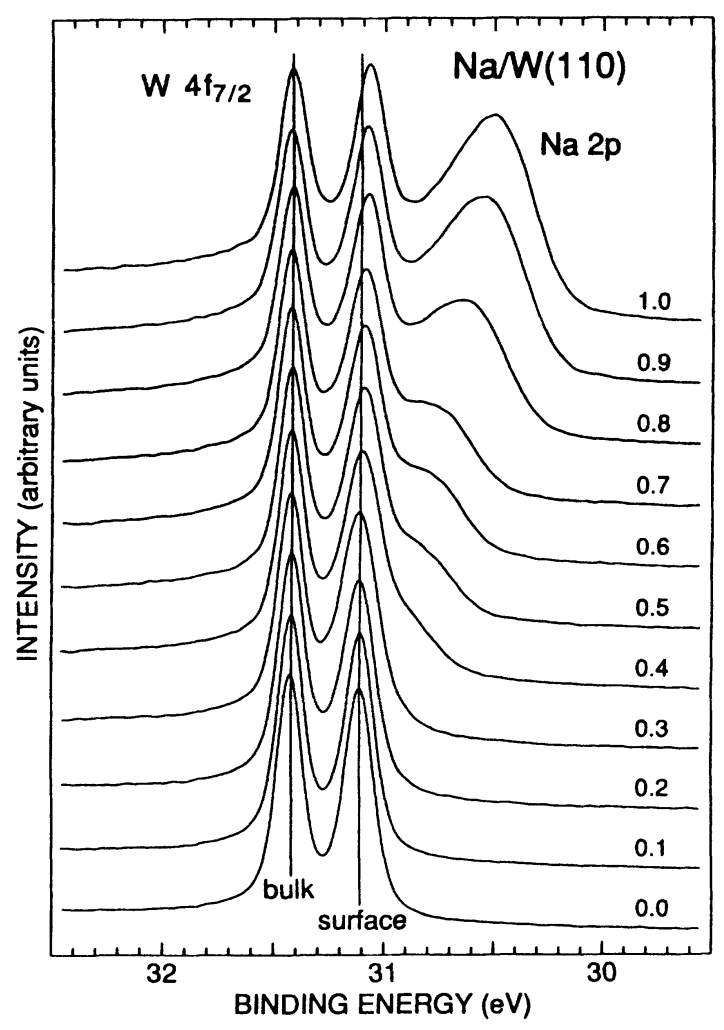

FIG. 7. W $4 f_{7 / 2}$ photoemission spectra from $\mathbf{W}(110)$ at $78 \mathrm{~K}$ adsorbed with $\mathrm{Na}$. Coverage is given as a fraction of saturation, defined here as $1.0 \mathrm{ML}$.

+96 and $+91 \mathrm{meV}$ for $\mathrm{Na}$ and $\mathrm{Rb}$, respectively. ${ }^{30}$ Such positive shifts are in accord with an alkali-substrate interaction of the type described by IT, ${ }^{5}$ whereby the dominant effect is the hybridization of alkali and adsorbate orbitals. In this context, the relatively larger positive shifts found for Ta are likely a result of the larger density of states of those metal.

Two other interesting aspects of the behavior shown in Fig. 8 are the absolute magnitude of the shifts and their turnover to decreasing and eventually negative values as saturation coverage is approached. It is important to keep in mind that the shifts in Fig. 8 are extremely small compared with those normally associated with unit changes in valence. Back donation, in which a substantial fraction of an electronic charge is presumed to return to the alkali atom as the coverage increases, would clearly be expected to produce changes in $\Delta_{s, b}$ that are large and positive at the very coverage where the observed shifts are instead small and start to decrease. The independent finding that the alkali-adsorbate-substrate vibrational stretch does not change with coverage ${ }^{31}$ is still further evidence that back donation is of little consequence. Both that result and these data, therefore, are incompatible with the basic notion of back donation contained in the Langmuir-Gurney model.

By contrast, the small change with coverage of the observed shifts is entirely compatible with the theory of IT, ${ }^{5}$ in which the change in the charge state of the adsorbate is negligible and coverage independent. The small nega- 


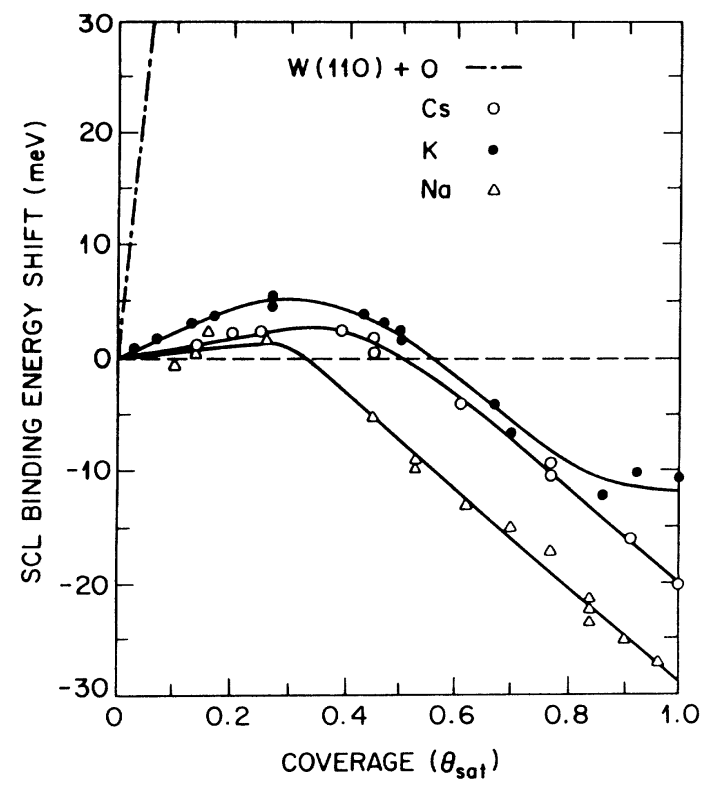

FIG. 8. Change in $\mathbf{W} 4 f_{7 / 2}$ surface-atom core-level (SCL) binding-energy shift produced by adsorbing $\mathrm{Na}, \mathrm{K}, \mathrm{Cs}$, and $\mathrm{O}$. Coverage is given as a fraction of saturation, defined here as 1.0 ML.

tive trends observed in Fig. 8 may be ascribed to additional final-state screening provided by the increasingly metallic adsorbate layer.

\section{DISCUSSION}

The photoemission data presented here serve to constrain the interpretation of the alkali-metal-substrate interaction in several specific ways.

(1) Evidence against the concept of back donation is obtained from both the coverage dependence of the lifetime width of the Cs $5 p$ core hole and the coverage dependence of the $\mathrm{W} 4 f$ surface-atom core-level shift. The results are, however, in good agreement with the calculations of IT, ${ }^{5}$ which predict charge clouds associated with the alkali adatoms that remain very similar at all coverages.

(2) Evidence supporting the formation of an alkalimetal $s$-like conduction band at high coverage is obtained from the magnitude of the singularity index of the alkali $5 p$ core level. This is in good agreement with the theory of Wimmer et al.$^{4}$ for Cs on W(100).

(3) Evidence supporting the concept that the charge cloud associated with each alkali atom at low coverage has strong alkali valence $s$ and $p$ character is obtained from the unprecedently increased magnitude of the outermost $p$ core-hole lifetime. Such $s-p$ hybridization was first proposed by Muscat and Newns ${ }^{8,9}$ and later reevaluated by IT. 5

Taken together, these experimental results point to a model in which the alkali charge resides in a cloud made up of strongly $s-p$ hybridized alkali character and located between the surface of the metal and the adsorbed alkali at all coverages. At higher coverage, the overlapping charge clouds form an alkali conduction band (metallization), which decreases the polarization of the adsorbate layer (depolarization). The overall charge state of the adsorbate atom does not significantly change with coverage, i.e., back donation is unimportant. The essential features of the Langmuir-Gurney model, ${ }^{1,2}$ therefore, are simply not realized.

Attempts to preserve the initially intuitively appealing concept of charge donation do not stand up to scrutiny.

(1) Benesh and King ${ }^{32}$ have calculated a positive shift of $190 \mathrm{meV}$ for substrate core levels when an alkali atom is brought up to the adsorbate position, keeping all charge distributions frozen. They then conclude that the much smaller measured shifts must be due to a fortuitous cancellation with a negative charge-transfer contribution, which arises when the frozen orbitals are allowed to relax. They do not consider the alternate process, namely, the formation of the hybridized charge cloud described by $\mathrm{IT},{ }^{5}$ which produces comparable negative shifts and accounts for other details of our observations.

(2) Pacchioni and Bagus ${ }^{33}$ carried out a calculation for a small $\mathrm{Cu}$ cluster with an external positive charge to represent a fully ionized alkali atom, obtaining a - 120meV shift for the core levels of cluster atoms next to the external charge. They then argue, in view of the stated uncertainty in their results of about $100 \mathrm{meV}$ due to other mechanisms, that their model provides an explanation of the measured shifts for the $\mathrm{W}$ core levels. As mentioned above, however, these shifts are actually much smaller and are positive. If these $\mathrm{Cu}$ cluster calculations are relevant to the $\mathrm{W}$ data, they must also account for the recent results in $\mathrm{Ta},{ }^{30}$ where the initial shifts are about +95 $\mathrm{meV}$ for atoms near the alkali adsorbates at low coverage, clearly outside the range of the theory. The inherently negative calculated charge-transfer shifts simply fail to account for the positive experimental shifts at low coverage.

(3) Scheffler et al. ${ }^{6}$ calculate charge distributions very similar to those of IT, $^{5}$ but they view the process as taking place in two steps, namely, charge transfer followed by the formation of a screening cloud. The orbitals that make up this cloud are not specifiable in their work. The intermediate charge-transfer state is not experimentally accessible. This two-step procedure is ad hoc and provides no insight into the nature of the state that is actually formed.

Throughout this paper, we have intentionally avoided using the terms "ionic" and "covalent" to describe the nature of the alkali-substrate interaction because neither one accurately describes the true situation. The process of charge donation in the Langmuir model has historically been referred to as an "ionic" interaction, but the resulting charge distribution bears no resemblance to that of an ionic crystal. Evidence for the absence of such charge donation and for the accumulation of charge between the alkali adsorbate and metal substrate atoms have both led to the use of the term "covalent," but the interaction between these atoms is very much weaker and much less directional than that implied by this description. The actual nature of the alkali-adatom-metalsubstrate interaction is, simply put, unique, and therefore not well characterized by conventional terminology. In this sense, then, at least part of the problem in describing 
the special nature of the alkali-adatom-substrate interaction has indeed been one of semantics. ${ }^{6}$

In summary, we have presented new evidence for a screening transition in alkali layers adsorbed on metal substrates, for a positive surface-atom core-level shift of the substrate at low adatom coverages, and for an enormously increased lifetime width of outermost alkaliadatom $p$ states. Our findings are compatible with a wide range of recent experimental results for alkali adsorption on a variety of substrates, ${ }^{30,31,34-38}$ all of which point to the interaction between the adsorbed alkalis and the substrate as being one that is not well described by charge donation. An interaction whereby the alkali adatoms retain their valence charge, with that charge becoming highly localized in the region between the adatom and the substrate, is much more appropriate.

\section{ACKNOWLEDGMENTS}

Photoemission research was carried out at the National Synchrotron Light Source, Brookhaven National Laboratory, which is supported by the Department of Energy, Division of Materials Sciences and Division of Chemical Sciences.
*Present address: Department of Physics, Utah State University, Logan, UT 84322.

${ }^{1}$ I. Langmuir, J. Am. Chem. Soc. 54, 2798 (1932).

${ }^{2}$ R. W. Gurney, Phys. Rev. 47, 479 (1935).

${ }^{3}$ D. M. Riffe, G. K. Wertheim, and P. H. Citrin, Phys. Rev. Lett. 64, 571 (1990).

${ }^{4}$ E. Wimmer, A. J. Freeman, J. R. Hiskes, and A. M. Karo, Phys. Rev. B 28, 3074 (1983).

${ }^{5}$ H. Ishida and K. Terakura, Rev. B 36, 4510 (1987); 38, 5752 (1988); H. Ishida, ibid. 38, 8006 (1988); 39, 5492 (1989); 40, 1341 (1989).

${ }^{6}$ M. Scheffler, Ch. Droste, A. F. Leszar, F. Máca, G. Wachutka, and G. Barzel, Physica B 172, 143 (1991).

7J. W. Gadzuk, Surf. Sci. 6, 133 (1967); B. N. J. Persson and H. Ishida, Phys. Rev. B 42, 3171 (1990).

8J. P. Muscat and D. M. Newns, Solid State Commun. 11, 737 (1972); J. P. Muscat and I. P. Batra, Phys. Rev. B 34, 2899 (1986).

${ }^{9}$ J. P. Muscat and D. M. Newns, Surf. Sci. 84, 262 (1979); J. Phys. C 7, 2630 (1974).

${ }^{10}$ N. D. Lang, in Physics and Chemistry of Alkali Metal Adsorption, edited by H. P. Bonzel, A. M. Bradshaw, and G. Ertl (Elsevier, New York, 1989), pp. 11-24.

${ }^{11} \mathrm{~J}$. Cousty, C. A. Papageorgopoulos, and R. Riwan, Surf. Sci. 223, 479 (1989).

${ }^{12}$ T. K. Sham and J. Hrbek, J. Chem. Phys. 89, 1188 (1988); M. L. Shek, J. Hrbek, T. K. Sham, and G.-Q. Xu, Phys. Rev. B 41, 3447 (1990).

${ }^{13}$ A. Hohlfeld, M. Šunjić, and K. Horn, J. Vac. Sci. Technol. A 5, 679 (1987).

${ }^{14}$ Unit coverage, $1 \mathrm{ML}$, here denotes first-layer saturation for the $W(110)$ surface, equal to $5.25 \times 10^{14} \mathrm{~cm}^{-2}$.

${ }^{15}$ S. Doniach and M. Šunjić, J. Phys. C 3, 285 (1970).

${ }^{16}$ A. G. Fedorus and A. G. Naumovets, Surf. Sci. 21, 426 (1970).

${ }^{17}$ G. K. Wertheim and D. N. E. Buchanan, Phys. Rev. B 43, 13815 (1991).

${ }^{18}$ R. L. Fink, P. N. First, and C. P. Flynn, Phys. Rev. B 38, 5839 (1988).

${ }^{19}$ T. A. Calcott, E. T. Arakawa, and D. L. Ederer, Phys. Rev. B
$18,6622(1978)$

${ }^{20}$ C.-O. Almbladh, A. L. Morales, and G. Grossmann, Phys. Rev. B 39, 3489 (1989).

${ }^{21}$ N. D. Lang and A. R. Williams, Phys. Rev. B 16, 2408 (1977).

22J. A. D. Matthews and Y. Komninos, Surf. Sci. 53, 716 (1975).

${ }^{23}$ D. L. Walters and C. P. Bhallah, Phys. Rev. A 4, 2164 (1971).

${ }^{24}$ D. M. Riffe, G. K. Wertheim, J. E. Rowe, and P. H. Citrin, Phys. Rev. B 45, 3532 (1992), and unpublished.

${ }^{25}$ G. K. Wertheim, D. M. Riffe, and P. H. Citrin, Phys. Rev. B 45, 8703 (1992).

${ }^{26}$ P. H. Citrin, G. K. Wertheim, and Y. Baer, Phys. Rev. B 16, 4256 (1977).

${ }^{27}$ P. H. Citrin, G. K. Wertheim, and Y. Baer, Phys. Rev. B 27, 3160 (1983).

${ }^{28}$ D. M. Riffe, G. K. Wertheim, and P. H. Citrin, Phys. Rev. Lett. 65, 219 (1990).

${ }^{29}$ C. Tréglia, M. C. Desjonquères. D. Spanjaard, Y. Lassailly, C. Guillot, Y. Jugnet, T. M. Duc, and J. Lecante, J. Phys. C 14, 3463 (1981).

${ }^{30}$ A. B. Andrews, D. M. Riffe, and G. K. Wertheim (unpublished).

${ }^{31}$ C. Astaldi, P. Rudolf, and S. Modesti, Solid State Commun. 75, 847 (1990).

${ }^{32}$ G. A. Benesh and D. A. King, Chem. Phys. Lett. 191, 315 (1992).

${ }^{33}$ G. Pacchioni and P. S. Bagus, Surf. Sci. 269/270, 669 (1992).

${ }^{34}$ R. Souda, W. Hayami, T. Aizawa, S. Otani, and Y. Ishizawa, Phys. Rev. Lett. 69, 192 (1992).

${ }^{35}$ B. Woratshek, W. Sesselman, J. Kuppers, G. Ertl, and F. Haberland, Phys. Rev. Lett. 55, 1231 (1985).

${ }^{36}$ D. Heskett, K.-H. Frank, K. Horn, H.-J. Freund, A. Baddorf, K.-O. Tsuei, and E. W. Plummer, Phys. Rev. B 37, 10387 (1988).

${ }^{37}$ K. Horn, A. Hohlfeld, J. Somers, Th. Lindner, P. Hollins, and A. M. Bradshaw, Phys. Rev. Lett. 61, 2488 (1988); H. Ishida, ibid. 63, 1535 (1989).

${ }^{38}$ R. Dudde, L. S. O. Johansson, and B. Reihl, Phys. Rev. B 44, 1198 (1991). 